\title{
Automation, teamwork, and the feared loss of safety
}

\author{
Air traffic controllers' experiences and expectations on current and future ATM systems
}

\author{
Åsa Svensson* \\ Department of Science and Technology, Linköping \\ University, Norrköping, Sweden \\ asa.e.svensson@liu.se \\ Camilla Forsell \\ Department of Science and Technology, Linköping \\ University, Norrköping, Sweden \\ camilla.forsell@liu.se
}

\author{
Jonas Lundberg \\ Department of Science and Technology, Linköping \\ University, Norrköping, Sweden \\ jonas.lundberg@liu.se \\ Niklas Rönnberg \\ Department of Science and Technology, Linköping \\ University, Norrköping, Sweden \\ niklas.ronnberg@liu.se
}

\begin{abstract}
In various control systems, automation is implemented to increase efficiency and safety. With increased automation, it becomes increasingly relevant to view the automation as a team member, rather than as a tool. In best cases, human-automation teamwork keeps workload within acceptable limits, increase situation awareness, and keeps the operator in the control loop. However, humanautomation teamwork will only flourish if the automation is developed with the human operator in mind. Therefore, investigations of the current experiences and expectations regarding automation and teamwork are important for the development of automation. Through a questionnaire among Air Traffic Controllers (ATCOs), the present study aims to investigate how ATCOs perceive automation and safety in current and future air traffic control systems and the importance of different teamwork factors for human-human and human-automation collaboration. The results indicate that the ATCOs believe that safety will increase in the future along with increasing automation as long as the automation is working as expected. The ATCOs expressed a fear of deskilling and losing situation awareness with automation, a fear associated with a new ATCO role of monitoring the system and take over when the automation fails. The results suggest that design for human-automation teamwork aspects that ATCOs value, such as adaptability or mutual performance monitoring, could be a way forward.
\end{abstract}

\section{CCS CONCEPTS}

- Human-centered computing $\rightarrow$ Human computer interaction (HCI); Human computer interaction (HCI); Interaction paradigms; Collaborative interaction; $\bullet$ HCI design and evaluation methods $\rightarrow$ User studies.

${ }^{*}$ Corresponding author.

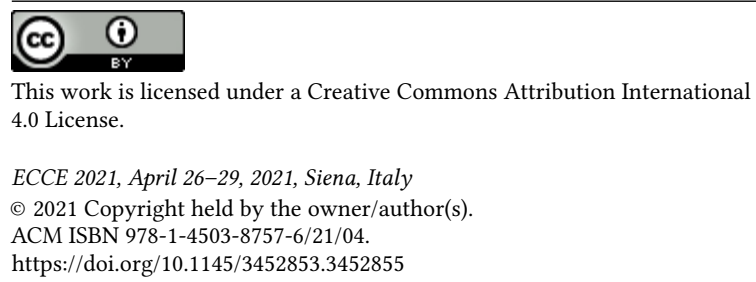

\section{KEYWORDS}

Teamwork, Automation, Safety, Air Traffic Control

\section{ACM Reference Format:}

Åsa Svensson, Jonas Lundberg, Camilla Forsell, and Niklas Rönnberg. 2021. Automation, teamwork, and the feared loss of safety: Air traffic controllers' experiences and expectations on current and future ATM systems. In European Conference on Cognitive Ergonomics 2021 (ECCE 2021), April 26-29, 2021, Siena, Italy. ACM, New York, NY, USA, 8 pages. https://doi.org/10.1145/3452853.3452855

\section{INTRODUCTION}

Safety-critical domains, such as Air Traffic Management/Control (ATM/ATC), railway traffic control, and nuclear power plants, are facing similar challenges at the moment, such as the demand for higher efficiency, more complex situations, and the need for increased safety. To be able to meet such challenges, higher levels of automation [1-3] are often seen as a requirement [4, 5]. Automation is introduced with the intent to keep workload within acceptable limits, reduce human errors, and increase Situation Awareness (SA) [6]. For example in ATM, automation should progressively enable increased Air Traffic Controller's (ATCO) productivity and airspace capacity [7], with the goal of an even more efficient industry. Therefore, various forms of advanced automation have been designed to reduce operator load and support performance [8-10]. While automation is introduced to increase safety, which is the fundamental basis in ATM [4], higher levels of automation might also introduce risks for decreased safety $[11,12]$. Higher levels of automation have historically been proven to negatively affect operator's own control performance since the operator is being removed from the control loop [13-15] which might result in loss of SA [14] among other things. This motivates new design studies to address those issues with automation in ATM/ATC. It is also important for other domains, such as for example the maritime domain, that looks at ATC for inspiration in their own automation work. If the current designs from ATC are copied to other domains then there is a risk that these concerns will follow.

Increased use of automation within any system is also dependent on user acceptance of the automation [16, 17]. Within the ATM industry, there has been a belief that new automation can be met with resistance and scepticism from the ATCOs [16, 18]. Various factors affect whether automation is accepted and used, including trust in automation [19,20], domain experience [21], age [22], and 
information requirements the user has regarding the automation [23], as well as cultural aspects, familiarity with computer usage, experiences with previous automation [24], job satisfaction [17], and usefulness of automation [25]. Moreover, with increased automation, it becomes relevant to include teamwork perspective on automation, rather than strictly viewing automation as a tool for the operator to use. This has been recognised in previous studies, which have suggested designing for human-automation teamwork [26] with automation designed in relation to proficient use by the ATCOs [17, 23], and for teamwork between operators [27]. However, few studies have recently addressed ATCOs' experience of current ATM systems. Therefore, this study explores operators' current experiences of teamwork and expectations of automation to better understand how automation can be implemented to support the work of the operator in relation to human-automation teamwork. This study uses ATM as a case. ATM is an interesting domain to study due to its exceptional safety record and on-going digitalization and implementations of automation. Exploring the topics of automation, human-human teamwork, and human-automation teamwork with over 200 operational ATCOs from six different countries has not, to our knowledge, been conducted in recent years. This study provides unique research and results about the operators' experiences and expectations. The results can be used for the design of automation which supports human-automation teamwork. Even though ATM has been used as a case, the results can be useful in other control domains as well which faces similar opportunities and challenges as the ATM industry [28-30]. In a collaboration with COOPANS (an international collaboration between the air navigation service providers of Austria (Austro Control), Croatia (Croatia Control), Denmark (Naviair), Ireland (Irish Aviation Authority), Portugal (NAV Portugal), and Sweden (LFV)), the topics of automation, safety, workload, SA, human-human teamwork, and human-automation teamwork were explored through a questionnaire consisting of statements with five-point Likert-scales, as well as free-text questions. The questionnaire was distributed to ATCOs in six countries.

The aim of the present study was to address the ATCOs' experiences of and expectations on automation in ATM systems. This up-to-date knowledge should be of interest when planning and designing automation in future ATM systems. More precisely the current study answered the following questions: 1) How do ATCOs perceive the grade of automation and the grade of safety in their current ATM systems and how do they believe the grade of automation and the grade of safety will change in the future? 2) What is the experienced workload and SA when working in current ATM systems and how do ATCOs believe workload and SA will change in the future? 3) Which teamwork attributes do ATCOs believe are of most importance when working with human colleagues compared to working with an automated system? 4) How do ATCOs view, qualitatively, their work relation with future automation?

\section{BACKGROUND}

Human factors literature predicts an increase in future traffic volume and, consequently, ATCO workload $[6,7,31]$. As a result of the increased complexity, more advanced automation is needed $[4,5]$ to maintain (or even increase) the present safety requirements. Automation can be defined as " a device or system that accomplishes (partially or fully) a function that was previously, or conceivably could be, carried out (partially or fully) by a human operator" [31]. It can be applied to different degrees or levels [1], from single decision steps to entire systems [1-3, 32-34]. For example, the automatic sorting of the flight strips on the electronic flight progress board could have one level of automation while the warning system within the board has another level of automation.

\subsection{Attitudes towards automation in ATM}

When investigating the automation acceptance threshold in relation to levels of automation, a survey found that most of the respondents approved of an automated tool that assisted them in organizing materials for decision-making [35]. However, comparatively few respondents in the survey approved of a tool that meant that the authority to make decisions shifted away from the operator. Human-automation teamwork could contribute to the operator's involvement in decision-making, especially while the operator is having the authority. Operators are cautious since experience has taught them to be cautious [36]. One reason for this could be that maintaining SA $[37,38]$ and decision-making for control action are two core cognitive tasks for the operators in ATM [39]. By introducing automation that intrudes on these core tasks, i.e. when the operator is prevented from making decisions and actions regarding traffic movements and separations, this means that the operator's role is fundamentally changing. Thereby, for many ATCOs, it appears to be difficult to accept new concepts with high-level automation and even more if so they cannot see the possible benefits of the new system [21]. In addition, it has been reported that ATCOs appeared reluctant to resign responsibility for decision making or control to the automation [40]. The ATCOs seemed to accept advisory automation, as long as they remained in the position of making decisions. The ATCOs also feared that future automation within ATM will include more monitoring tasks and, thereby, greater risks of mistakes when taking control if the automation would fail. Thus, the willingness to accept automation appears to be task-dependent and also dependent on the role of automation, its actions and intensions [35]. Further, ATCOs who are generally more satisfied with their work situation are more accepting of higher levels of automation than less satisfied ATCOs [35]. ATCOs have also reported that if automation is to be used, standardizations are important to guarantee safety [25]. Finally, in ATM contexts, teamwork factors for human-human teamwork have been identified as central to successful work.

\subsection{Teamwork}

A team can be seen as a set of two or more people who interact with each other in a dynamic way, and who adaptively work toward a common goal [41], and satisfying teamwork between team members can enhance performance [27]. However, with the introduction of automation, at least at higher levels where automation acts more as an agent, comes also the introduction of human-automation teamwork for the operator to stay in the control loop [32]. Therefore, investigations of the current experiences and expectations 
regarding automation and teamwork are relevant in the development of human-centred automation. Salas, Sims and Burke [42] provided an overview of empirically supported claims regarding teamwork, summarized in the Big Five model of teamwork factors for effective teamwork. The Big Five model contains five core components: team leadership, mutual performance monitoring, backup behaviour, adaptability, and team orientation, and three coordinating mechanisms: shared mental models, mutual trust, and closed-loop communication [42]. The Big Five model contains attributes that are necessary for teamwork to be effective, regardless of the type of team and domain [42]. It aims to cover the many different essential aspects of teamwork and offers a holistic framework, rather than focusing more narrowly on input (information given to the team) and output (actions taken by the team). (See, for example, the model by McGrath [43].) The Big Five model has been applied in various contexts, such as in learning environments [44], in army peace-keeping teams [45], in project teams [46], and for fighter pilots teamwork experiences [47]. Regarding ATCO teamwork, it was found that mutual trust was the most important teamwork factor followed by adaptability and shared mental models [27].

\section{METHOD}

A questionnaire was distributed as a web form through ATM managers to ATCOs in six different countries, including ATC sites such as area control, approach control, and tower control: 249 licensed ATCOs (200 male and 44 female, 5 choose not to disclose gender), from six different countries (Austria (34), Croatia (24), Denmark (31), Ireland (71), Portugal (19), and Sweden (67)), took part in the study. The respondents' median experience was 18 years, with a minimum of 0 years and a maximum of 39 years, a mean of 16.78 years. Age was divided into three groups: 40 years old or younger (88 respondents), between 40 and 49 years (109 respondents), and 50 years or older ( 52 respondents). The questionnaire was voluntary, and respondents were not incentivized. It consisted of a total of 139 questions and statements split into five sections (experiences and expectations of current and future current ATM system, humanhuman teamwork, human-automation teamwork, and demography). The Big Five model was used as a foundation for the human-human teamwork and human-automation teamwork questions. However, in ATC, the team orientation factor can be taken for granted, since selected and trained professionals and the ability to work in a team is a prerequisite [27]. This aspect was, therefore, excluded from the present study.

Through pre-tests with ATCOs, the time required to answer the questions was estimated to about 20 minutes, a limit suggested by COOPANS for practical purposes. Likert scales [48] ranging from 1 $=$ 'very low' or 'not at all important' to $5=$ 'very high' or 'extremely important' were used as well as optional free-text sections. Respondents were free to move back and forth between questionnaire sections and change their responses if needed. Statistical analysis was performed using non-parametric tests, e.g. Wilcoxon Signedrank test $(Z)$ due to the ordinal nature of the data. In addition, Kruskal Wallis $\mathrm{H}(\chi 2)$ post-hoc tests were applied to the data for making one-way ANOVA on ranks. Friedman's 2 ways ANOVA $(\chi 2)$ was applied to analyse the teamwork factors. Bonferroni correction for multiple comparisons was used when appropriate. To analyse the difference between the respondents' current experiences and the respondents' expectations for the future, the current experience rating score was subtracted from the rating score of the expectations. The possible difference was investigated in relation to country, gender, age groups, and endorsement. Cronbach's Alpha [49] has not been applied due to the number of test items (i.e. three statements for each teamwork factor). Moreover, since the questionnaire uses more than one construct, Cronbach's Alpha for the test as a whole is not reported due to the variety of questions which can inflate the value of Cronbach's Alpha. Free-text answers, which were optional for the respondents to answer, were analysed and here presented by an overall thematic analysis [50,51].

\section{QUANTITATIVE ANALYSIS}

This section provides the quantitative results regarding the grade of automation, workload, SA, safety, human-human teamwork, and human-automation teamwork, see Figure 1. The respondents (n $=244$, five stated "Don't know" and were excluded) expected a significantly higher grade of automation $(Z=-8.68, p<0.001)$ for the future $($ median $=4$, mean $=4.05$, range 1-5) compared with today (median 3; mean $=3.31$, range $1-5)$. Post-hoc tests showed significant differences between countries $(\chi 2=20.49, \mathrm{p}<0.001)$. Ireland had a smaller increase of expected automation compared to Sweden ( $p=0.035)$, Austria $(\mathrm{p}=0.019)$, and Portugal $(\mathrm{p}=0.021)$. The respondents ( $\mathrm{n}=237$, twelve stated "Don't know" and were excluded) believed that workload would be higher $(\mathrm{Z}=-2.12, \mathrm{p}=$ 0.034 ) in the future (median $=3$, mean $=3.46$, range $1-5$ ) compared with today (median $=3$, mean $=3.33$, range $1-5$ for today). The respondents ( $\mathrm{n}=243$, six stated "Don't know" and were excluded) believed that their SA would decrease $(Z=-8.40, p<0.001)$ in the future (median $=3$, mean $=3.44$, range $1-5$, ) compared with today $($ median $=3$, mean $=4.22$, range $2-5)$. There was an effect of countries for the change of grade of $\mathrm{SA}(\chi 2=17.31, \mathrm{p}=0.004)$. However, post-hoc tests did not reveal any significant pairwise differences between countries. The respondents $(n=236$, thirteen stated "Don't know" and were excluded) believed that safety would decrease $(Z=-8.01, p<0.001)$ in the future $($ median $=4$, mean $=3.1$, range $1-5$ ) compared with today (median $=5$, mean $=4.51$, range 1-5). There was an effect of countries for the change in believed grade of safety $(\chi 2=19.95, \mathrm{p}<0.001)$, where Sweden had a greater decrease of safety compared to Portugal $(\mathrm{p}=0.001)$.

For the analysis of the teamwork factors, the three statements within each teamwork factor were summed, see Figure 2 and Table 1. Regarding human-human teamwork, Friedman's 2 ways ANOVA showed a significant difference in ranking of the teamwork factors $(\chi 2(6)=293.7, \mathrm{p}<0.001)$. Post-hoc tests showed that adaptability was significantly ranked as a more important teamwork factor compared to all other teamwork factors. Team leadership was ranked as the least important teamwork factor for human-human teamwork. Adaptability got a median score of 13 , while team leadership got a median score of 10 . However, no teamwork factors got a lower score than ten. The rankings of human-automation teamwork factors were also significantly different $(\chi 2(6)=461.1, \mathrm{p}<0.001)$. Post-hoc tests showed that adaptability was significantly ranked as a more important teamwork factor compared to all other teamwork factors except for mutual performance monitoring which 


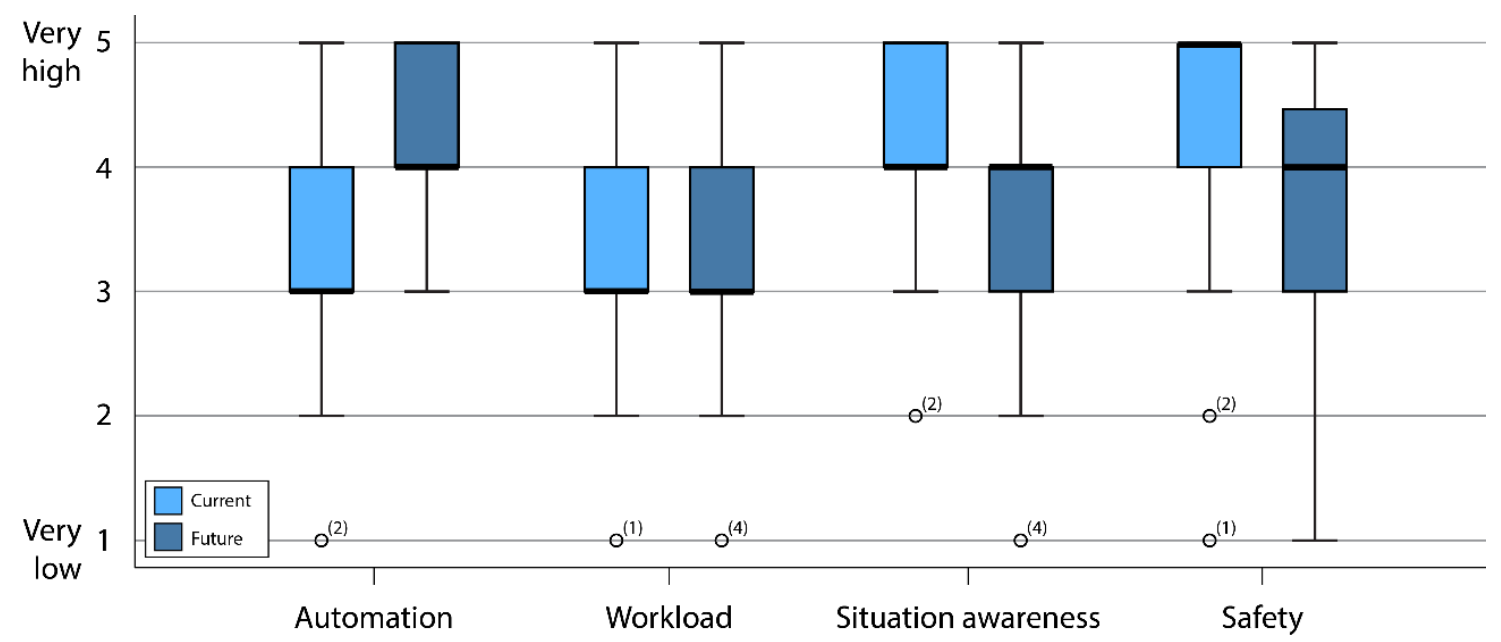

Figure 1: Compared with today, the participants believe the grade of automation will be higher in the future, that workload will stay the same, that SA will decrease in the future, and that safety will decrease in the future. The numbers by the circles represent the number of outliers.

Table 1: The scores for all teamwork factors, including a total score where the scores for human-human teamwork and humanautomation teamwork has been added together.

\begin{tabular}{llll}
\hline Teamwork factor & $\begin{array}{l}\text { Human- } \\
\text { humanteamwork }\end{array}$ & Human-automationteamwork & Total score \\
\hline Adaptability & 13 & 12 & 25 \\
Team leadership & 10 & 9 & 19 \\
Mutual performance monitoring & 12 & 11 & 23 \\
Backup behaviour & 11 & 7 & 18 \\
Shared mental models & 11 & 10 & 21 \\
Closed-loop communication & 12 & 10 & 22 \\
Mutual trust & 12 & 9 & 21 \\
\hline
\end{tabular}

did not have a significant difference from adaptability. Backup behaviour was ranked as the least important teamwork factor for human-automation teamwork. Adaptability got a median score of 12, while backup behaviour got a median score of seven. However, no teamwork factors got a lower score than seven. When adding the median scores of human-human teamwork and human-automation teamwork, adaptability was seen as the most important teamwork while backup behaviour was the least important factor. For humanautomation teamwork, team leadership received the same score as mutual trust. However, since mutual trust was seen as more important in human-human teamwork, mutual trust was reported as the fourth most important teamwork factor together with shared mental models. Mutual performance monitoring was the second most important factor, with a similar score for both human-human and human-automation (12 and 11).

\section{THEMATIC ANALYSIS OF FREE-TEXT ANSWERS}

The analysis of free-text answers showed that the ATCO views on human-human teamwork differed from their views on (future) human-automation teamwork. Human-human teamwork in ATM was expressed as important overall. Notably, the degree of importance depends on the workload, the working position (which role the controllers have, and in which sector the controllers are working), and the situation (traffic load, time of day, etc.). Furthermore, working in standard teams (the same ATCOs working in the same constellation of teams during each work shift) were expressed to improve teamwork. Based on these comments it can be assumed that having standard teams could also lead to trust between the team members. Trust was expressed to improve capacity, safety, and SA.

In addition to having standard teams with strong trust, the respondents also believed that verbal communication and mutual performance monitoring are of importance between the human team members. Mutual performance monitoring was also the second most important factor for human-automation teamwork, according to the quantitative answers.

In contrast to the quantitative analysis, it was expressed that safety can increase with higher degrees of automation and that any "safety net or tool that can enable the controller to focus more on decision making at active tasks will be a benefit". However, too 


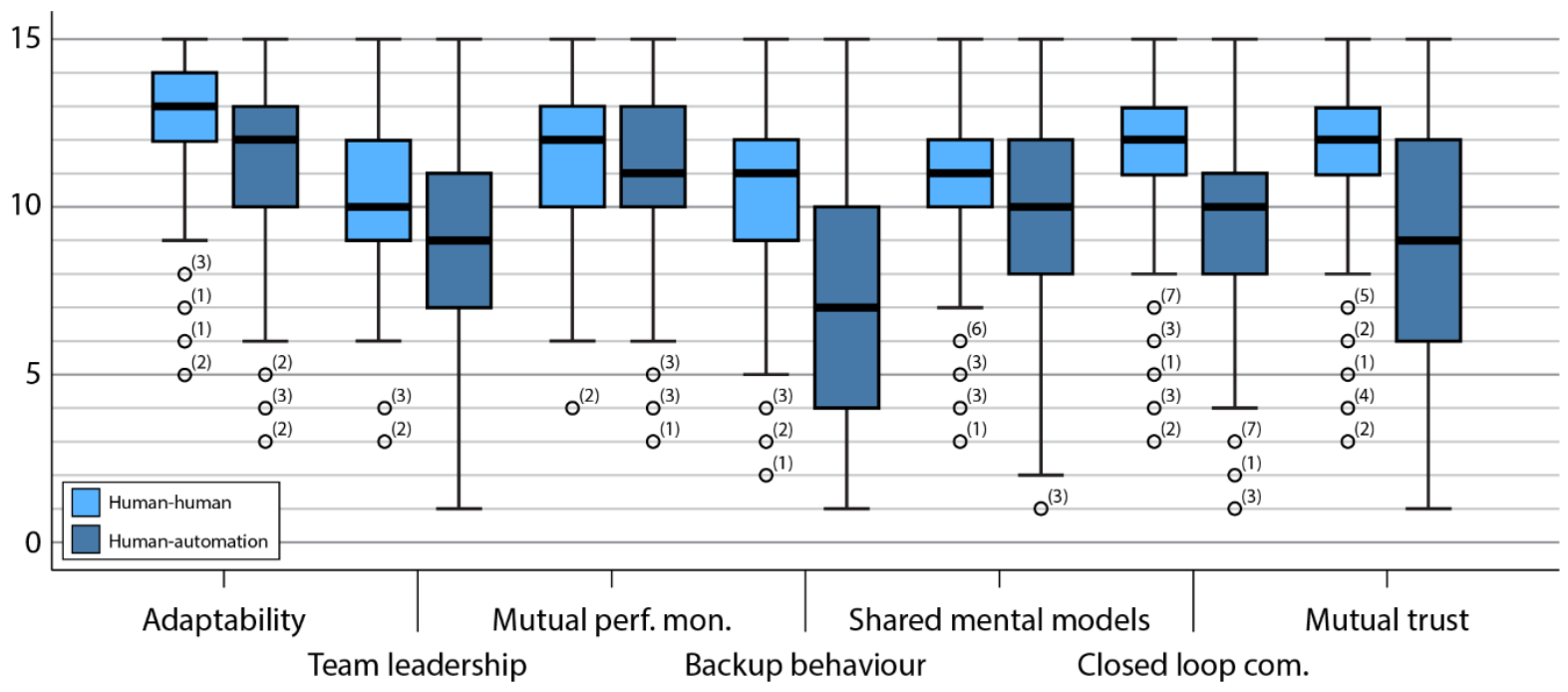

Figure 2: For both human-human teamwork and human-automation teamwork, adaptability was the most important teamwork factor. Team leadership was the least important teamwork factor for human-human teamwork while backup behaviour was the least important for human-automation teamwork.

"much automation in decision making/action-taking is worrying to a controller as it distances them from the sector and decisions/actions taken". Thus, the respondents were pro automation as long as the operator remains in control and has the responsibility: "It is crucial that any automation does not take responsibility away from the controller".

Even though the respondents expressed that safety will increase along with automation, they saw potential risks with implementing automation. The respondents expressed that ATCOs in the future will more passively monitor the traffic situation and while monitoring the automation, compared with today when they are actively controlling the traffic. It was expressed that ATCOs in the future will function as a backup to the automated systems (instead of the other way around), i.e. as a form of a one-way performance monitoring where only the ATCO is monitoring and having knowledge about the automation. These thoughts lead to a fear of potential risks, that the ATCO will be out-of-the-loop. It was also expressed that SA would decrease in the future if there would be more automation, especially if the ATCOs would serve as a backup system. Automation was seen as useful only during the circumstances when everything goes as planned (with increased safety as well). However, the respondents were worried about what would happen when the automation does not work, fails, or if anything unpredicted happens. Then, automation was seen as a hazard. In such situations, the ATCO will not "be able to handle such amount of traffic as a fully automated system" or be able to intervene when not prepared as "Automation for sure reduces situation awareness", and that "automation is good until there's too much of it and you lose your situation awareness". Furthermore, automation could cause problems since it might function as "a sleeping pill if the system normally takes care of the traffic itself”. ATCOs expressed a fear of deskilling, that a loss of ATCO operational and practical skills, would be "a real risk with automation. I think better support for
ATCOS is more important than "taking over" tasks from the ATCO. Today, it is too easy to disregard warnings and/or acknowledge visible warnings without analysis". Deskilling would also negatively affect the ATCO's ability to intervene and take control from the automation if necessary because the "controller must be actively involved to keep skill levels and to catch the dropped ball if the system fails. And it will”. It was stated that in such situations, control would be even more vulnerable if the ATCO functions as a backup system to the automation.

Moreover, respondents expressed a need for new requirements and policies regarding safety and security to enable humanautomation teamwork in ATM, e.g. "it must be said that capacity and more efficient use of airspace does not only depend on system, automation etc. Things could be done differently already today, but it would require new rules and regulations, politics etc... Appropriate training and procedures for working with automation and staffing will be necessary to keep situational awareness, "but once proper training and practice come in to play then situational awareness will improve". ATCO skills need to be trained for and balanced in relation to automation: "The more monitoring tasks the controller will be assigned to and used to, the less skills to take initiative to actions when needed. What will happen when the automatic systems fail and the controller no longer can act because he is no longer trained for this task?".

There was a concern regarding trust in the automation. The ATCO might trust the automation too much and be biased towards it, and might "become overconfident, careless" when working with the automation. There was also a fear that automation might be implemented to lower costs, and that this "will lead to an over trust in automated solutions. It is not given it will be safer". With increased automation, the ATCO might not question the actions of the automation or might be put out-of-the-loop by not gathering 
enough information about the situation. Therefore, what a controller needs "from an automated system is to be able to trust its solutions and not have to second guess the supplied information". It can be assumed that the ATCO would have an increased workload due to being out-of-the-loop if the ATCO has to intervene in tasks the automation should perform. In addition, it was expressed that the workload will be kept at a high level, as "controllers will control more aircraft with increased automation". The respondents expressed that the ATCO must be involved in decision making and in control to stay in the loop and to not lose practical skills. For that, relevant information is needed: "I don't need a fully automated system, I need relevant info". To keep workload at a reasonable level and to create trust for the automation, the respondents did not want to have more automation but would prefer if the automation provided the information needed. Therefore, available information "needs to be prioritized so only relevant information is used (too much information will create information overload)". It was stated that to support the human operator and not hinder performance, the automation needs to be even better than a human replacement: "the automation [that] is replacing my planner it needs to perform equally good or better".

\section{DISCUSSION}

The results show that a belief in increased automation is associated with a feared loss of safety. The respondents believe that the level of automation will increase in the future (similar to $[4,6]$ ) and that automation only will be beneficial as long as the automation supports the controller (similar to what was found 8 years ago in [35]). The analysis indicates that this fear of safety loss is associated with a specific design of automation where human-automation teamwork is characterised by each having a specific role. For instance, humans being a safety net for fallible automation was seen as a hazard whereas the automation as a safety net to humans was seen as a benefit. The ATCOs expressed that safety will increase along with automation, however, only as long as nothing unexpected happens. According to the qualitative results, there was a belief that the automation would be controlling the traffic but with the human operator still having legal responsibility. The respondents expressed a fear of shifting the control from the ATCO to the automation, leaving the ATCO as a backup system to the automation and intervene only when the automation is unable to manage the situation. If so, it would be a one-way performance monitoring instead of mutual performance monitoring as one of the central teamwork factors. This shift in roles is associated with a fear of the ATCO being outside of the control loop [13,32] and not being able to intervene if the automation would fail. Being out-of-the-loop would lead to decreased SA and, thus, decreased safety. To have the legal responsibility while only monitoring the situations and leaving the decision making to an automation can lead to disastrous consequences [29] when the human operator has to intervene for any reason. Even though automation increases with the aim to aid the ATCO, workload is expected by the respondents to stay at the same level. This might be due to the predicted increase in traffic load and complexity [7]. Increased automation opens up possibilities for more complex situations and, hence, the workload is unchanged. However, as the levels of automation increases in various systems, problems of vigilance may increase, also expressed in the free-text answers. The more reliable the system becomes, the less work there is for a person to do, and the mind begins to wander [52]. To be able to work with the automation, the results in the present study suggest that the ATCOs want to know what is happening around them (mutual performance monitoring). This means maintaining SA, knowing what the team members are doing, being in control, and having adaptability. The results were the same regarding automation. It should be designed to support mutual performance monitoring for the operator to know what the automation is doing. The automation should be seen as a support, as a team member which has the necessary teamwork attributes which are important in the domain. In ATM systems, mutual performance monitoring should be an automation attribute, having knowledge about what everyone in the team is doing (and also share its own intentions) together with closed-loop communication and adaptability. In addition, as in line with previous research [53] the ATCOs stated in the free-text answers, ATCO skills need to be trained for in relation to automation.

\subsection{Future work}

The results from the present study are indicative of what ATCOs within COOPANS experience and believe about current and future ATM systems. Even though the overall sample size was high, the number of respondents from each country varied greatly. Therefore, comparisons between countries have not been made due to the uneven distribution of respondents. Future research should compare attitudes between countries, age, and endorsement as well. The results found in the present study provide a clear foundation for further research regarding beliefs for the future ATM systems. The results indicate a fear for having automation taking control from the ATCO, leaving the ATCO to only monitor and intervene when the automation is unable to manage the situation. This calls for more research regarding how the automation can be designed to support SA and to keep the human in the loop as long as the human has the legal responsibility. The results suggest that design research could focus on incorporating the teamwork factors that the ATCOs believed are central to human-automation collaboration. Additionally, investigating how benefits of automation are communicated would also be relevant, since the introduction of automation might affect the operator's attitude [54]. The questionnaire used in the present study can also be used in other control domains (e.g. railway traffic control, aviation, nuclear power plants etc.) to get a deeper understanding of operators' (users') view on automation, workload, SA, safety, and teamwork. Finally, it is important to make a follow-up study few years from now, to explore any changes in operational ATCOs' opinions regarding current and future ATM systems.

\section{CONCLUSIONS}

This study indicates that human-automation teamwork is central to the respondents' view of increased automation. Automation as a safety net was seen as a benefit, whereas ATCOs as a backup system was seen as a hazard. The analysis suggests that this change of roles could be associated with the fear of safety loss. ATCOs feared deskilling of operational skills and being out-of-the-loop as their 
work tasks would be to only monitor the situation and backup the automation when the automation is unable to manage the situation, i.e. a form of a one-way performance monitoring instead of having mutual performance monitoring which the ATCOs ranked as one of the most important teamwork factors for human-automation teamwork. The fact that this view is present in the ATM community, with its outstanding safety record, should be a wake-up call for the control system design community. It was also found that ATCOs valued certain human-automation teamwork factors to the same extent as human-human teamwork factors. One way forward could be to break out of the role of humans as a backup when automation fails. This role division is particularly ironic since automation is sometimes introduced to reduce errors from human failures. The results in the present study suggest that these roles become particularly troublesome if automation extends to carry out tasks, rather than providing a stronger basis for decision-making. Based on the analysis it is suggested to establish design approaches that include teamwork aspects such as adaptability or mutual performance monitoring.

\section{ACKNOWLEDGMENTS}

The authors would like to thank all the respondents who answered the questionnaire. A direct special thanks to COOPANS who assisted in distributing the questionnaire. The present study was funded by the Swedish Transport Administration, and the LFV Air Navigation Services of Sweden, via the project Amplify Teamwork with Automation.

\section{REFERENCES}

[1] Sheridan, T.B., W.L. Verplank, and T.L. Brooks. Human/computer control of undersea teleoperators. in Proceedings of the International Conference on Cybernetics and Society. 1978. Tokyo-Kyoto, Japan: IEEE.

[2] Endsley, M.R., Level of Automation Forms a Key Aspect of Autonomy Design. Journal of Cognitive Engineering and Decision Making, 2018. 12(1): p. 29-34.

[3] Kaber, D.B., Issues in human-automation interaction modeling: Presumptive aspects of frameworks of types and levels of automation. Journal of Cognitive Engineering and Decision Making, 2018.

[4] SESAR, Air Transport Framework - The Current Situation. 2006: Brussels, Belgium.

[5] SESAR, European ATM Master Plan Level 3 - Implementation Plan. 2017: Brussels, Belgium.

[6] SESAR, European ATM Master Plan. 2015: Brussels, Belgium.

[7] SESAR, A proposal for the future architecture of the European airspace. 2019 Brussels, Belgium.

[8] Martinie, C., P. Palanque, A. Pasquini, M. Ragosta, E. Rigaud, et al. Using complementary models-based approaches for representing and analysing ATM systems' variability. in 2nd International Conference on Application and Theory of Automation in Command and Control Systems-ATACCS'12. 2012. IRIT Press.

[9] Fürstenau, N., Virtual and Remote Control Tower. 2016: Springer.

[10] Bagassi, S., Augmented and Virtual Reality in the Airport Control Tower: The RETINA concept. 2017.

[11] Kontogiannis, T. and S. Malakis, Cognitive Engineering and Safety Organization in Air Traffic Management. 2017.

[12] Strauch, B., Ironies of Automation: Still Unresolved After All These Years. IEEE Transactions on Human-Machine Systems, 2017. 48(5): p. 419 - 433.

[13] Endsley, M.R. and E.O. Kiris, The out-of-the-loop performance problem and level of control in automation. Human Factors, 1995. 37(2): p. 381-394.

[14] Endsley, M.R. and D.B. Kaber, Level of automation effects on performance, situation awareness and workload in a dynamic control task. Ergonomics, 1999. 42(3): p. 462 $-492$.

[15] Baxter, G., J. Rooksby, Y. Wang, and A. Khajeh-Hosseini. The ironies of automation: still going strong at 30? in Proceedings of the 30th European Conference on Cognitive Ergonomics. 2012. Edinburgh, United Kingdom: ACM.

[16] Hilburn, B. and M. Flynn. Air traffic controller and management attitudes toward automation: an empirical investigation. in Air Traffic Management Reserach and Development Seminar. 2001. Santa Fe, USA.

[17] Bekier, M., Automation acceptance in air traffic management, in Deptartment of Aviation. 2013, New South Wales: Sydney, Australia.
[18] Borst, C., C. Westin, and B. Hilburn. An Investigation into the Use of Novel Conflict Detection and Resolution Automation in Air Traffic Management. in SESAR Innovation Days. 2012. Braunschweig, Germany.

[19] Lee, J.D. and K.A. See, Trust in Automation: Designing for Appropriate Reliance. Human Factors, 2004. 46(1): p. 50-80.

[20] Mirchi, T., K.-P. Vu, J. Miles, L. Sturre, S. Curtis, et al., Air Traffic Controller Trust in Automation in NextGen. Procedia Manufacturing, 2015. 3: p. 2482-2488.

[21] Eurocontrol, Age, Experience and Automation in European Air Traffic Control Survey in the ECAC Area. 2004: Brussels, Belgium.

[22] Thompson, R.C. and L.L. Bailey, Age and attitudes in the air traffic control specialist workforce: an initial assessment. 2000, Federal Aviation Administration: Oklahoma City, USA.

[23] Parasuraman, R., J. Duley, and A. Smoker, Automation tools for controllers in future air traffic control. The Controller, 1998.

[24] Eurocontrol, Attitudes towards future automation concepts: A litterature review. 2000: Brussels, Belgium.

[25] Nijhuis, H., S. Buck, C. Kelly, P. Goillau, C. Fassert, et al., RHEA. Role of the Human in the Evolution of ATM systems. Final report. 1999.

[26] Norman, D., Design, business models, and human-technology teamwork: As automation and artificial intelligence technologies develop, we need to think less about human-machine interfaces and more about human-machine teamwork. Research-Technology Management, 2017. 60(1): p. 26-30.

[27] Svensson, A.., U. Ohlander, and J. Lundberg, Design implications for teamwork in ATC. Cognition, Technology \& Work, 2019.

[28] Banks, V.A. and N.A. Stanton, Keep the driver in control: Automating automobiles of the future. Applied ergonomics, 2016. 53: p. 389-395.

[29] Endsley, M.R., Testimony to the United States House of Representatives: Hearing on Boeing 737-Max8 Crashes. 2019.

[30] Naser, J., Guidance for the Design and Use of Automation in Nuclear Power Plants. 2005, Electric Power Research Institute: California, USA.

[31] Parasuraman, R., T. Sheridan, and C. Wickens, A model for types and levels of human interaction with automation. IEEE Transactions on Systems, Man, and Cybernetics - Part A: Systems and Humans, 2000. 30(3): p. 286 - 297.

[32] Endsley, M.R. and E.O. Kiris, Out-of-the-Loop Performance Problems and the Use of Intermediate Levels of Automation for Improved Control System Functioning and Safety. Human Factors: The Journal of the Human Factors and Ergonomics Society, 1995. 37(2): p. 381-394.

[33] Miller, C. and R. Parasuraman, Beyond Levels of Automation: An Architecture for More Flexible Human-Automation Collaboration. Proceedings of the Human Factors and Ergonomics Society Annual Meeting, 2003. 47(1): p. 182

[34] Wickens, C., Automation Stages \& Levels, 20 Years After. Journal of Cognitive Engineering and Decision Making, 2018. 12(1): p. 35-41.

[35] Bekier, M., B.R. Molesworth, and A. Williamson, Tipping point: The narrow path between automation acceptance and rejection in air traffic management. Safety science, 2011. 50(2): p. 259-265.

[36] Hopkin, V.D. and J.A. Wise, Human Factors in Air Traffic System Automation. Automation and human performance: Theory and applications, 1996: p. 192.

[37] Endsley, M.R., Toward a Theory of Situation Awareness in Dynamic Systems. Human Factors, 1995. 37(1): p. 32-64.

[38] Stanton, N.A., P.M. Salmon, G.H. Walker, E. Salas, and P.A. Hancock, State-ofscience: situation awareness in individuals, teams and systems. Ergonomics, 2017. 60(4): p. 449-466.

[39] Eurocontrol, Model for Task and Job Descriptions of Air Traffic Controllers. European Air Traffic Control Harmonisation and Integration Programme. 1996: Brussels, Belgium.

[40] Hilburn, B., Conflict Resolution Assistant Level 2 (CORA2) Controller Assessments: Final Report. 2000.

[41] Salas, E., T.L. Dickinson, S.A. Converse, and S.I. Tannenbaum, Toward an understanding of team performance and training, in Teams: Their training and performance, R.W. Swezey and E. Salas, Editors. 1992, Ablex: Norwood, NJ. p. 2-29.

[42] Salas, E., D.E. Sims, and C.S. Burke, Is there a "big five" in teamwork? Small group research, 2005. 36(5): p. 555-599.

[43] McGrath, J.E., Social psychology: A brief introduction. 1964: Holt, Rinehart and Winston.

[44] Kay, J., N. Maisonneuve, K. Yacef, and P. Reimann. The big five and visualisations of team work activity. in 8th International Conference on Intelligent Tutoring Systems, ITS 2006. 2006. Jhongli, Taiwan: Springer.

[45] Duel, J., Teamwork in action: Military teams preparing for, and conducting Peace Support Operations. 2010, Tilburg University: Haag, Netherlands.

[46] van Roosmalen, T.M., The development of a questionnaire on the subjective experience of teamwork, based on Salas, Sims and Burke's" the big five of teamwork" and Hackman's understanding of team effectiveness. 2012, The Norwegian University of Science and Technology: Trondheim, Norway.

[47] Ohlander, U., Towards Enhanced Tactical Support Systems, in Artificial Intelligence Lab. 2016, Högskolan i Skövde Skövde, Sweden.

[48] Fink, A., How to conduct surveys: a step-by-step guide. 5th ed. 2013, London, UK: Sage Publisher. 
[49] Cronbach, L.J., Coefficient alpha and the internal structure of tests. psychometrika, 1951. 16(3): p. 297-334

[50] Creswell, J.W., Research Design: Qualitative, Quantitative, and Mixed Methods Approaches. Vol. 3. 2009, Thousand Oaks, California, US: Sage Publications, Inc.

[51] Richards, L., Handling qualitative data: A practical guide. 2014: Sage.

[52] Norman, D.A., The Human Side of Automation, in Road Vehicle Automation 2, G. Meyer and S. Beiker, Editors. 2015, Springer International Publishing: Cham. p.
73-79.

[53] Drogoul, F. and P. Palanque, Education and Performance in Aviation: Realising and Sustaining Benefits. 2019, Eurocontrol: Brussels, Belgium.

[54] Bekier, M. and B.R.C. Molesworth, Altering user'acceptance of automation through prior automation exposure. Ergonomics, 2017. 60(6): p. 745-753. 